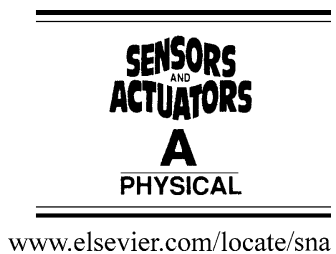

\title{
A dynamic tactile sensor on photoelastic effect
}

\author{
Venketesh N. Dubey ${ }^{\mathrm{a}, *}$, Richard M. Crowder ${ }^{\mathrm{b}, 1}$ \\ ${ }^{a}$ Bournemouth University, School of Design, Engineering \& Computing, Talbot Campus, Fern Barrow, Poole BH12 5BB, UK \\ ${ }^{\mathrm{b}}$ University of Southampton, Intelligence, Agents, Multimedia Group, School of Electronics and Computer Science, Southampton SO17 1BJ, UK
}

Received 19 September 2005; received in revised form 23 December 2005; accepted 23 January 2006

Available online 28 February 2006

\begin{abstract}
Certain photoelastic materials exhibit birefringent characteristics at a very low level of strain. This property of material may be suitable for dynamic or wave propagation studies, which can be exploited for designing tactile sensors. This paper presents the design, construction and testing of a novel dynamic sensor based on photoelastic effect, which is capable of detecting object slip as well as providing normal force information. The paper investigates the mechanics of object slip, and develops an approximate model of the sensor. This allows visualization of various parameters involved in the sensor design. The model also explains design improvements necessary to obtain continuous signal during object slip. The developed sensor has been compared with other existing sensors and experimental results from the sensor have been discussed. The sensor is calibrated for normal force which is in addition to the dynamic signal that it provides from the same contact location. The sensor has a simple design and is of a small size allowing it to be incorporated into robotic fingers, and it provides output signals which are largely unaffected by external disturbances. (C) 2006 Elsevier B.V. All rights reserved.
\end{abstract}

Keywords: Dynamic sensor; Slip sensor; Photoelastic sensor; Shear sensing

\section{Introduction}

The human hand is adaptive in forming a variety of object grasps and in most cases the grasp is optimal i.e. the object is stable within the grasp and the forces applied to the object are the bare minimum. This capability can be attributed to the dexterity of the human hand, coupled with the use of the human senses and versatile processing power of the human brain. However, at localized hand-level this can be considered to be achieved by increasing the gripping force to arrest any ensuing object slip, thus maintaining the minimum gripping force. This simple technique can be implemented in a mechanical system if the force at the fingertip and the object slip can be detected simultaneously by sensors. This can be also useful in implementing object grasp by robotic end effectors, to minimize the power consumption and to avoid excessive gripping force over the grasped object without causing any damage to it. Hence, the conditions of gentle grasp can be achieved if the object is grasped with a small force and

\footnotetext{
* Corresponding author. Tel.: +44 1202 965986; fax: +44 1202965314

E-mailaddresses: vdubey@bmth.ac.uk(V.N.Dubey),rmc@ecs.soton.ac.uk (R.M. Crowder).

${ }^{1}$ Tel.: +4423 8059 3441; fax: +44238059 2865
}

the gripping force is increased when the object starts to slip. This requires a sensor for detecting object slip. Also in some cases of object manipulation, detection of slip may be necessary to cause sliding motions of fingers over the grasped object surface [1]. The need for slip detection of object has long been recognized for prosthetic applications [2] and has been considered useful in object manipulation [3]. However, it has also been reported that detection of slip is 'difficult to achieve' and often shadowed by external disturbances $[4,5]$.

Many techniques have been employed to detect object slip, however, none have compared or even remotely close to the sensitivity and robustness of human slip sensing capabilities. For robotic applications this has been achieved by a number of different techniques such as interpretation of tactile-array data, interpretation of touch-sensor data or by employing dedicated slip sensors [6]. A basic method of slip sensing was based on the 'lift and try' technique, where a change in motor current was monitored to assess the quality of the grip. This technique had obvious difficulties of differentiating gripping force from interaction force and had to start every time with a very small gripping force to reach to a large force when the object did not slip. Some other approaches [7] include a sapphire needle protruding from a sensor surface and touching the slipping object to generate vibrations, which in turn produces voltage from a 
piezoelectric crystal or from a permanent magnet and coils as a measure of object slip. Optical interrupt-type slip sensors have been designed with slotted roller [8] which interrupts an optical path passing across it, giving an indication of slip. Though this sensor gives a good indication of the speed and direction of slip the obvious disadvantages are poor slip resolution and the jamming of the roller. Additionally, if the sensor is used for grasping, the rolling contact at the object surface will tend to slip the object and stable grasp cannot be achieved.

In order to emulate the slip sensing capability of the natural hand, a number of pressure sensing sites have been created over the sensing surface. As the object slips, a change in pressure detected by sensors gives an indication of slip, however, it is difficult to differentiate slip of object with applied force changes to the sensing sites. In a slight deviation from the conventional approaches, Kyberd and Chappell [9] have developed a slip sensor based on forced oscillations, which detects the sound produced by vibrations using a microphone. Eghtedari and Morgan [10] have used photoelastic material and a solidstate camera with a detailed processing to infer the object slip. With the rapid development of VLSI chip fabrication technology many slip sensors have been designed as sensing arrays. A slip sensor using a piezoelectric bimorph element has been developed by Nishihara et al. [11]. The sensor is sensitive to dynamic stress which detects the instant of first slip as well as contact and release of an object. However, the sensor is easily influenced by vibrations, for which vibration absorbers are used. Holweg et al. [12] have used rubber based tactile matrix sensor to detect slip, which rely on the elasticity of the rubber but the response of the sensor is slow with non-linear characteristics and hysteresis. Of various available sensing technologies, many recent developments in slip sensing employ the sensing arrays than one-point contact sensors [13-17]. Although the research on slip sensing has emphasized the need for detection of incipient slippage for stable grasping and dexterous manipulation [18-20], such localized slip detection is often compounded by external disturbances. The sensor described in this paper obviates many of the above problems and is capable of detecting slip as well as the normal force from the same contact location. The sensor is based on the photoelastic effect and it produces continuous signal during object slip. The signals are so strong that they do not require any amplification, leaving it largely immune to the external disturbances. Experiments conducted with the sensor are reported.

\section{Mechanics of slip and photoelasticity}

In context of grasping and manipulation of objects, slip may be regarded as the relative movement of one object surface over another when they are in contact. The relative motion may be of any form ranging from simple translational to a combination of translational and rotational motions. Mechanically, slip can be thought to be shearing of two object surfaces in contact, in all possible forms of motions. Visually it may be easier to detect relative movement between two slipping objects, however, it is difficult to exactly analyze various material and geometrical interactions in terms of physical behavior of the contacting sur-

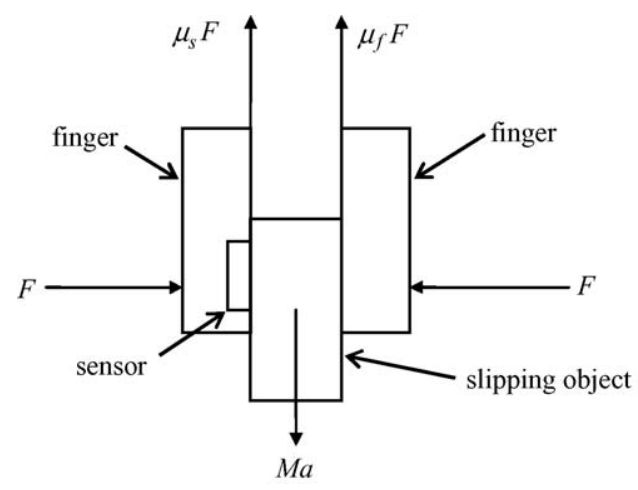

Fig. 1. A slipping object with system of forces.

faces during slip. Starting from the rest situation, the contact surfaces form a pressure distribution which cannot be predicted accurately (this is due to the random irregularity of the contacting surfaces) and when the object slips, this distribution is disturbed; the contact pressure however remains the same. How the pressure distribution changes, largely depends on the elastic nature of the objects, slip rate, surface roughness and the geometric boundary conditions. The change in pressure distribution, however, does not provide any detectable information (as opposed to the vision systems) about the object slip. In tactile sensing, the associated side effects such as acoustic sound produced during slip, vibration of the slipping surface or the relative movement between contact surfaces can only be exploited to detect the object slip.

The force experienced by an object slipping between two fingers is shown in Fig. 1.

The two fingers provide a force to hold the object stationary. It will slip if forces applied on the object including those due to gravity and acceleration are larger than the frictional forces, in which case,

$M a>F\left(\mu_{\mathrm{f}}+\mu_{\mathrm{s}}\right)$

Object will not slip if,

$M a \leq F\left(\mu_{\mathrm{f}}+\mu_{\mathrm{s}}\right)$

where $\mu_{\mathrm{f}}, \mu_{\mathrm{s}}$ are the coefficients of friction between the finger/sensor and the object and $a$ is the vector sum of acceleration due to gravity together with that supplied by the inertia. To prevent the object slipping, the applied finger force $(F)$ needs to be controlled so that the condition shown by Eq. (2) is always satisfied.

The schematic of the proposed sensor is shown in Fig. 2 where light emitted by a source is passed through optical fibers to the

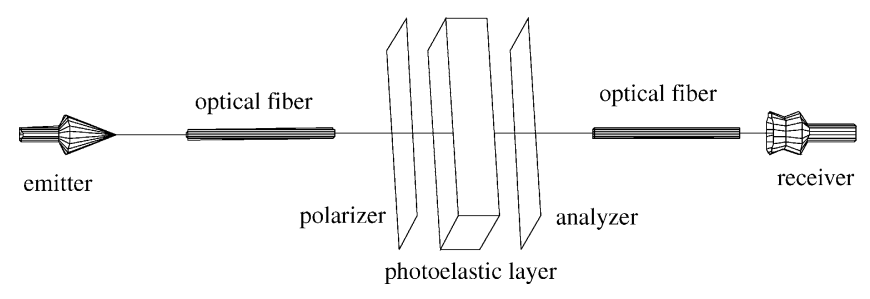

Fig. 2. Key features of the sensor. 


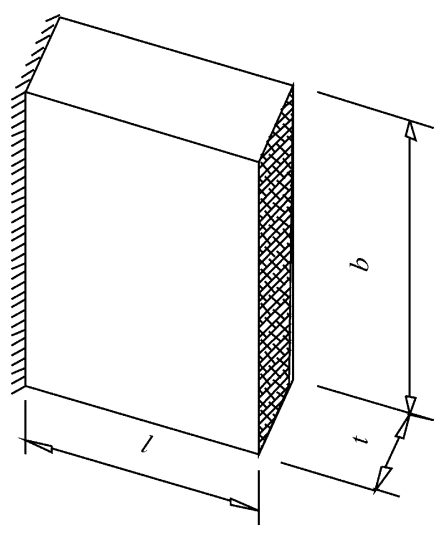

Fig. 3. Sensor dimensions and the boundary conditions.

photoelastic layer located between a polarizer and an analyzer. On entering the stressed transparent photoelastic material, the polarized light is split into two components which vibrate in the two perpendicular planes of principal stresses. The velocity of propagation of each component of light differs from that in the unstressed material by an amount which depends on the magnitude of the principal stresses. Consequently, the two component rays emerge from the material out of phase. The difference in phase depends on the difference between two principal stresses, the wavelength of light, thickness of the material in the direction of propagation of light, and the stress optical coefficient of the material. The two rays emerging from the material are received by an analyzer, which only transmits the components of two rays in its plane of polarization causing a change in the intensity of light at the receiver.

Fig. 3 shows the boundary conditions and dimensions of the photoelastic layer used in the sensor design. The layer has a fixed boundary forming a cantilever beam. The shaded portion forms the sensing area. The $l, b$ and $t$ are the length, breath and the thickness of the layer, respectively.

Under the action of the applied normal force and the shearing force for the case shown in Fig. 1, the free body diagram of the sensing material is shown in Fig. 4, where $\sigma_{x}$ is the normal stress along $x$-direction and $\tau_{x y}$ is the shear stress in $x y$-plane. The counter couple developed in the material is represented by the shear stresses $\tau_{y x}$ (opposite to $\tau_{x y}$ ). The normal and shear stresses are expressed as:

$$
\begin{aligned}
\sigma_{x} & =\frac{F}{A} \\
\tau_{x y} & =\frac{\mu_{\mathrm{s}} F}{A}
\end{aligned}
$$
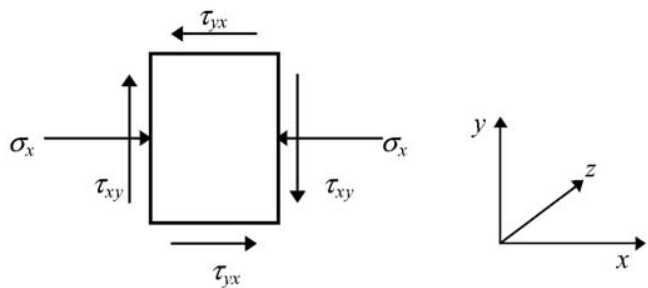

Fig. 4. Modeling of the sensing medium. and,

$A=b \times t$

where $F$ is the applied fingertip force, $A$ the contact area of the sensor and $\mu_{\mathrm{s}}$ is the coefficient of friction between the sensor-object surfaces. This is a condition of plane stress in $x y$ plane with negligible $\sigma_{y}$ component. The magnitude of principal stresses $\sigma_{1}$ and $\sigma_{2}$ (with $\sigma_{y}=0$ ) is given by [21]:

$\sigma_{1}=\frac{\sigma_{x}}{2}+\frac{1}{2} \sqrt{\sigma_{x}^{2}+4 \tau_{x y}^{2}}$
$\sigma_{2}=\frac{\sigma_{x}}{2}-\frac{1}{2} \sqrt{\sigma_{x}^{2}+4 \tau_{x y}^{2}}$

and the orientation $(\alpha)$ of the principal stresses is given by the angle between $x$-axis and the direction of the maximum principal stress $\left(\sigma_{1}\right)$ as:

$\alpha=\frac{1}{2} \sin ^{-1}\left\{\frac{2 \tau_{x y}}{\sqrt{\sigma_{x}^{2}+4 \tau_{x y}^{2}}}\right\}$

These stresses are represented in Fig. 5.

These relations are valid for semi-infinite solids but the sensor material considered here is a photoelastic layer which has finite dimensions so these relations are only an approximation for this application. Nevertheless, these relations give an idea of dependence of $\sigma_{1}, \sigma_{2}$ on the normal and the shear stresses over it. The intensity of light $\left(I_{0}\right)$ emerging out of the analyzer is expressed in terms of principal stress difference as [22]:

$I_{\mathrm{o}}=I_{\mathrm{i}} \sin ^{2} 2 \theta \sin ^{2}\left\{\frac{\pi t\left(\sigma_{1}-\sigma_{2}\right) c}{\lambda}\right\}$

where $I_{\mathrm{i}}$ is the incident intensity of light, $\theta$ the angle between plane of principal stress $\left(\sigma_{1}\right)$ and the plane of polarization, $t$ the thickness of the layer in the direction of light propagation, $\lambda$ the wavelength of the light used and $c$ is the stress optical coefficient (which is a known property of the material). The angle $\theta$ is shown in Fig. 6, where light enters the polarizer along the optical axis $\mathrm{O}$ (perpendicular to the plane of the paper). Light emerging from the polarizer has amplitude represented by the vector $\left(A_{\mathrm{p}}\right)$ along the axis of polarizer. When this polarized light enter the stressed photoelastic material, it splits into two component vectors $\left(A_{\mathrm{p} 1}\right.$ and $\left.A_{\mathrm{p} 2}\right)$ along the planes of principal stresses. At analyzer (whose axis is shown to be perpendicular

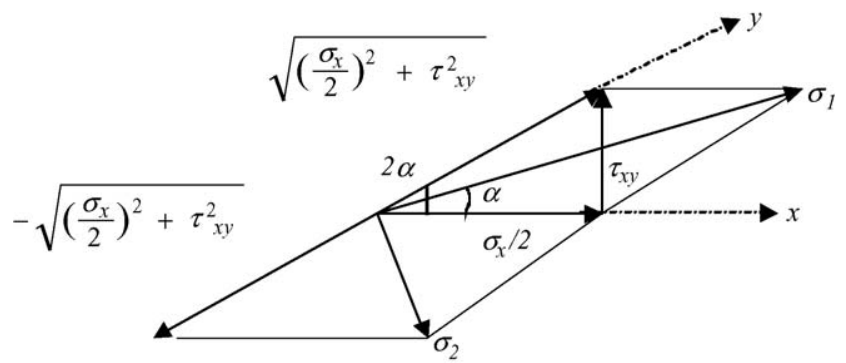

Fig. 5. Principal stresses and principal planes. 


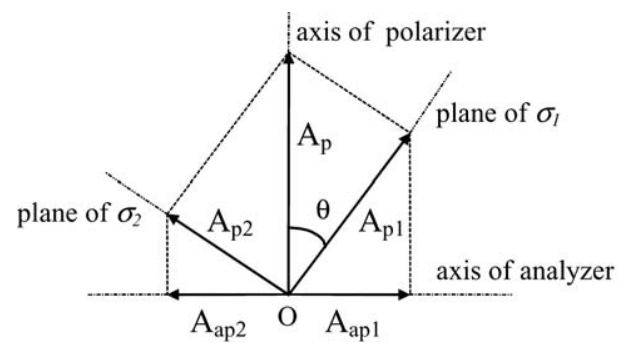

Fig. 6. Light representation in crossed polarizers.

to the axis of the polarizer), the components of these two vectors are taken along the axis of analyzer $\left(A_{\mathrm{ap} 1}\right.$ and $\left.A_{\mathrm{ap} 2}\right)$, which are out of phase according to the orientation of the principal planes. Thus the resultant vector is smaller, changing the intensity of the emerging light. Angles $\alpha$ and $\theta$ govern the sensitivity and dynamic range of the sensor.

Eq. (9) suggests that change in intensity of light at the receiver may be obtained only if there is a change in the value of $\sigma_{1}, \sigma_{2}$, other parameters being constant. Considering slip of an object, the system of stresses is changed at the very start of the slip due to sudden energy imparted to the photoelastic material and so is the case in reverse order when the slip is terminated. But during the slip with constant rate, the stresses remain constant. Thus no slip signal is available. However, the sensor could provide continuous slip signal if the slip motion can be partially converted into transverse motions to change the system of stresses. The key to achieve this is to improve the surface characteristics of the slip-surface of the photoelastic layer so that it can set up a system of vibration whenever an object slips over it.

\section{Slip sensor design}

In an attempt to produce continuous slip signals during object slippage the top surface of the photoelastic layer, which forms the slip surface, was changed with different geometry and materials as shown in Fig. 7, and tested experimentally. It has been found that the sensor did not provide continuous slip signals, since the projected edges of the sensor or the changed contact
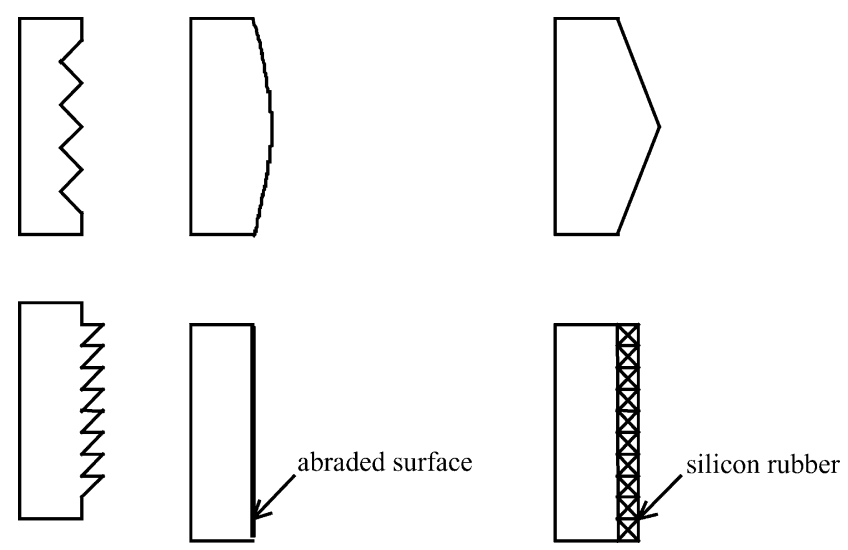

Fig. 7. The non-enhancing slip surfaces. surfaces in each case stuck to the slipping object at the constant slip rate.

If sensor's edges are partially loaded by the slipping object and are just making contact with the object during slip, vibration could be generated as reported in [19]. However, in the present study the object size was bigger than the sensor size which covers the entire surface area of the sensor, thus the sensor produced slip signals only during start and termination of the slip. Even abrading the slip surface and changing the top surface with silicon rubber were found to be unworkable. However, when the top surface was replaced by a metallic surface, it was found that the sensor produced continuous slip signal when the object slipped over it and the signal was found to be a function of the slip rate. This behavior was exhibited because of the self-excited vibration induced between the two contacting surfaces.

The phenomena of self-excited vibration due to dry friction have been observed when one object surface slides over the other. The process is caused by the fact that the frictional force does not remain constant as a function of velocity. It decreases as the relative velocity increases, giving rise to a negative damping to sustain the vibration of the system [23]. When the object surfaces are in contact and stationary, the relative velocity between the point of contact and the sliding surface is zero. When one surface starts sliding, a relative velocity is set and the frictional force acting at the interface displaces the point of contact which changes the relative velocity, which in turn changes the frictional force. When the relative velocity of the point of contact increases (i.e. when the second object moves against the sliding object) the magnitude of frictional force is reduced, as a result the amplitude of displacement decreases and so is the relative velocity of the point of contact. This increases the magnitude of frictional force and eventually increase the amplitude of displacement and the whole cycle repeats itself setting the system to vibrate. For such a vibration to take place the relative velocity must not cease, also the characteristic of vibration so exhibited is very much dependent on the material of the contacting surfaces which governs the change in frictional force with change in relative velocity. A complete theoretical analysis of the sensor based on frictional characteristics of different materials is available in [24], where it has been shown how vibration can be sustained and how the sensor can provide continuous signals at a constant slip rate.

The enhanced sensor is shown in Fig. 8, where a thin strip of stainless steel of $0.3 \mathrm{~mm}$ thickness was attached to the photoelastic layer. The final size of the sensing element was

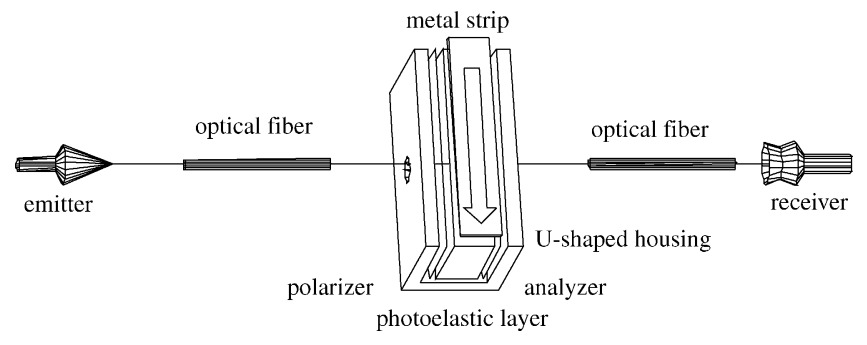

Fig. 8. The enhanced sensor. 


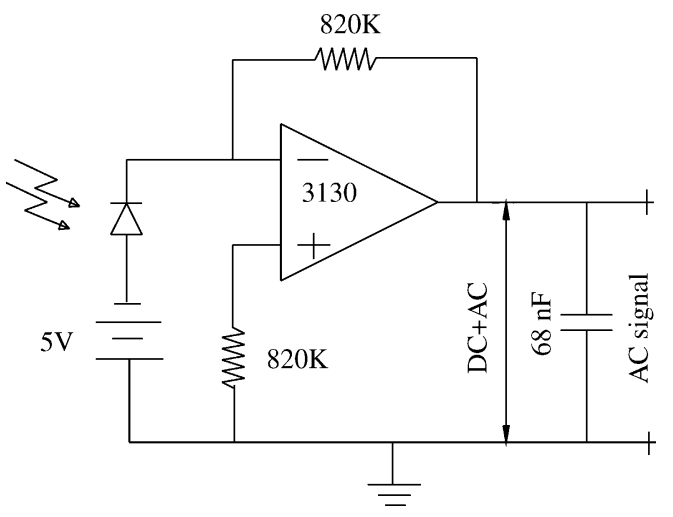

Fig. 9. The optical receiver and the filter used in the experiments.

$9 \mathrm{~mm} \times 5 \mathrm{~mm} \times 3 \mathrm{~mm}$, with a sensing area of $9 \mathrm{~mm} \times 3 \mathrm{~mm}$. The metallic strip was glued to the photoelastic layer (which forms the slipping surface) in such a way that it did not pre-stress the photoelastic material and with the same care the photoelastic layer was glued to a U-shaped body to house the sensor (Fig. 8). Care was also taken to place the polarizer/analyzer sheets in the U-shape in crossed mode so that it did not stick to the photoelastic layer. The optical fibers were fixed to the two arms of the sensor-body close to the slipping surface on two close-fit through holes. The arrow on the sensing area shows the direction of object slip and should be kept perpendicular to the optical fiber alignment to have the photoelastic effect [25]. The light source used is a high radiance emitter of peak spectral output of $850 \mathrm{~nm}$ and the receiver is a PIN photodiode to match the emitter. The single stage detection circuit used in this experiment is shown in Fig. 9.

The output from the amplifier contains both the normal force as well as the slip signals. When this signal is filtered, an ac signal is available which gives measure of slip and when the slip signal is subtracted from the main signal a dc signal is available (this has been achieved digitally in the software). This provides absolute information on the normal force.

\section{Experimental results}

The test-rig used for conducting experiments with the developed sensor is a three link articulated finger [26], shown in Fig. 10. A small dc servomotor controls slip of the object in vertical direction under gravity. The experiment uses objects of different mass and materials to compare the resulting slip signals. These test objects are a well machined aluminum block of average surface roughness $0.8 \mu \mathrm{m}$ and a plastic block of average surface roughness $0.5 \mu \mathrm{m}$. These objects do not have a surface roughness which can be exploited for the detection of slip. The objects are allowed to slip over the sensor surface under gravity and the applied force from the fingertip. The finger contact surface has a silicon rubber pad to provide a better closed-loop control of the object slip [27].

Fig. 11(a) shows the initial condition of signals as seen on an oscilloscope when no object is held. The upper plot in all the following figures (Figs. 11-13) is the dc (normal force) signal while the lower is the ac (slip) signal in a time frame of $50 \mathrm{~ms} / \mathrm{div}$. In order to see the smaller slip rates, the ac signal has been set at $20 \mathrm{mV} / \mathrm{div}$ while due to the initial offset of the dc signal, it has been set at $2 \mathrm{~V} /$ div. Since the dc signal has been set at a high voltage, small change in the signal cannot be seen in the plots. Fig. 11(b) shows the change in signals when a plastic block of $155 \mathrm{~g}$ is slipping. Due to the applied force, the dc signal is seen to increase from the previous value and the ac signal is seen to be perturbed. The variation exists throughout the timerange indicating that the slip signal is available continuously. The object slip rate in this case was $2.7 \mathrm{~mm} / \mathrm{s}$.

When the same object is allowed to slip at a lower rate $(0.72 \mathrm{~mm} / \mathrm{s})$, the amplitude as well as the frequency of the signal is seen to decrease, Fig. 12(a). The same experiment was repeated with an aluminum block of $105 \mathrm{~g}$ slipping at a rate of $0.61 \mathrm{~mm} / \mathrm{s}$, Fig. 12(b). Other conditions being the same, it can be seen that for approximately the same slip rate as in Fig. 12(a) (for the plastic block), the amplitude of the slip signal is higher for the metallic object as compared to the plastic object. It can

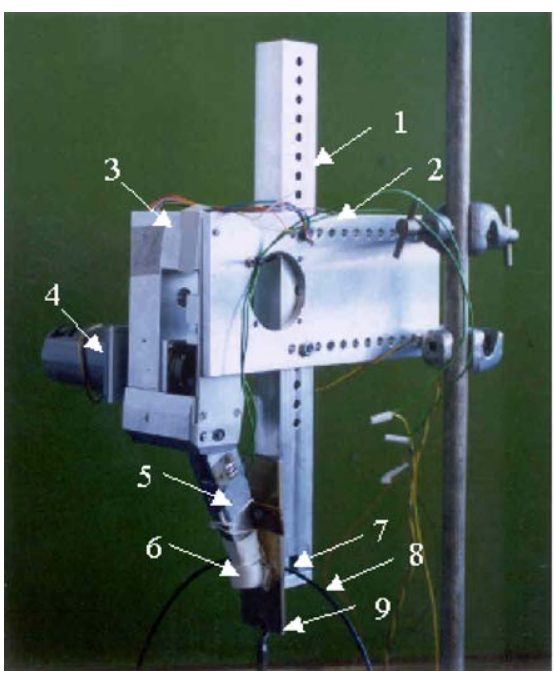

1. Adjustable support with sensor housing

2. Adjustable support plate

3. Finger mechanism housing

4. Finger drive motor

5. Articulated finger

6. Silicon fingertrip

7. The housed slip sensor

8. Optical fiber

9. Aluminum block (slipping object)

Fig. 10. The experimental set-up. 


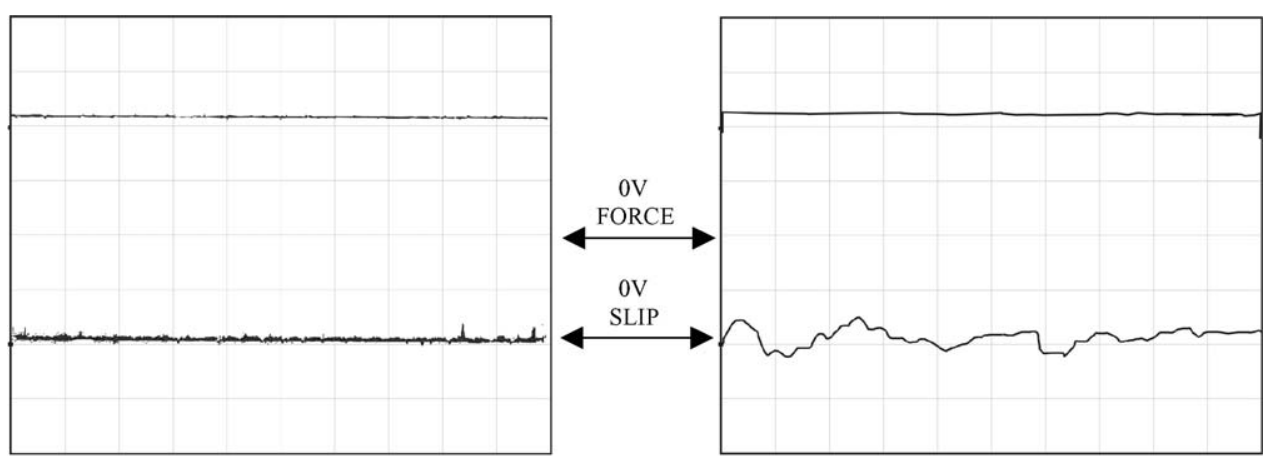

(a)

(b)

Fig. 11. ( $a$ and b) Initial conditions of slip and normal force signals.

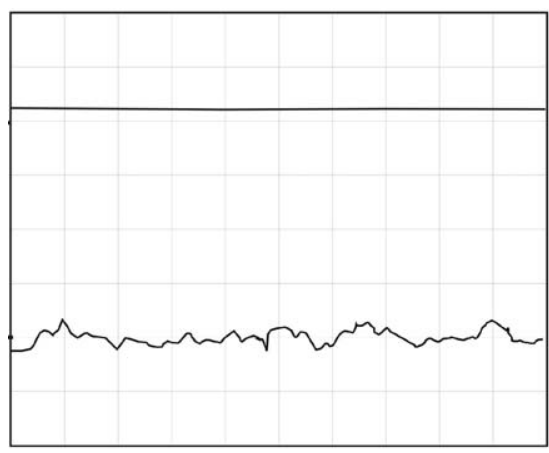

(a)

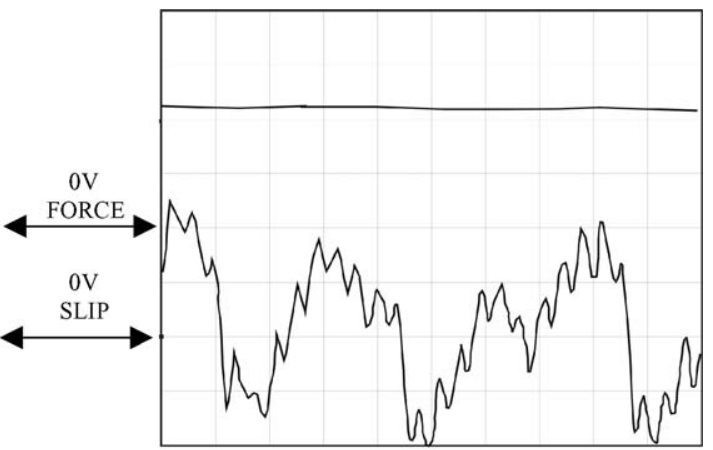

(b)

Fig. 12. ( $a$ and b) Slip signal for different materials at the same slip rate.

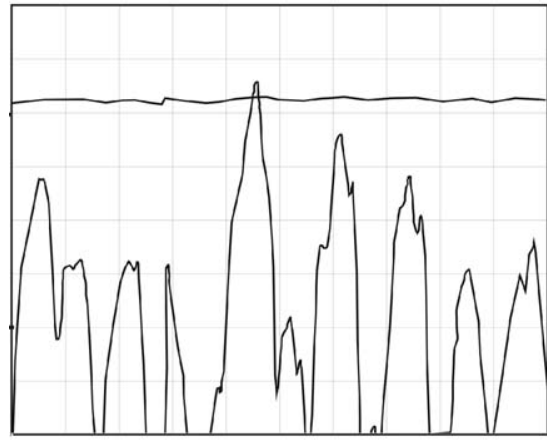

(a)

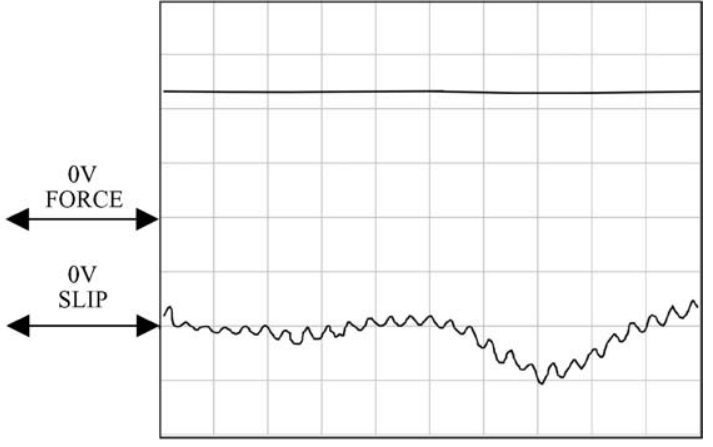

(b)

Fig. 13. ( $a$ and b) Slip signal for the same materials at different slip rates.

be concluded that the slip signal has high amplitude with metallic objects than with non-metallic objects at the same slip rate, since the friction-velocity relations are different for different materials.

Fig. 13(a) shows the signals obtained when the aluminum block slips at $3 \mathrm{~mm} / \mathrm{s}$. It can be seen that the amplitude, in this case is very high which also affects the applied force signal (upper plot), since the signal has both ac and dc components as seen on the oscilloscope. The slip signal is seen to be irregular and the frequency of the signal is found to be higher than the previous case in Fig. 12(b), means that for the same material at different slip rates the sensor provides different signals. The sensor is found to be capable of detecting slip rates as small as $0.1 \mathrm{~mm} / \mathrm{s}$ for certain metallic objects as can be seen from
Fig. 13(b). In all the above cases a change in dc signal provides the normal force information. Due to high initial offset, the dc signal cannot be easily differentiated in these plots for different forces applied to the object. However, the sensor has been calibrated for the normal force to fully characterize it. Further, the sensor has been tested against external disturbances by imparting artificial jerks and shocks to the test rig. It has been found that the sensor is immune to such disturbances and the signals remain spike-free.

\section{Sensor calibration for normal force}

The sensor has been calibrated for the normal force. The plot of applied force against voltage (which is proportional to the 


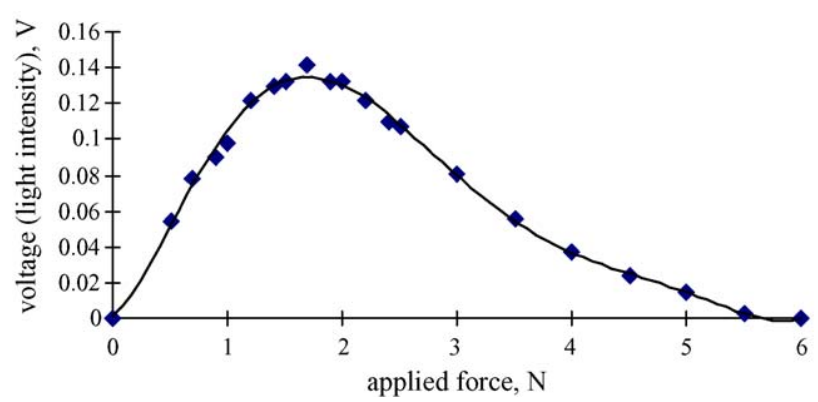

Fig. 14. The sensor calibration graph for the normal force.

light intensity at the receiver) is shown in Fig. 14. It can be seen that the variation closely follows the sine-squared function as has been predicted theoretically [22]. For the present sensor the voltage increases up to a force of $1.7 \mathrm{~N}$, after that the voltage drops to the initial condition providing a total force range of $6 \mathrm{~N}$. The sensor can be used until $1.7 \mathrm{~N}$ for absolute applied force measurement; however, the full range of $6 \mathrm{~N}$ can be exploited by suitably processing the signal to track the increasing or the decreasing trend of the voltage.

A limitation of the sensor, as is true in case of any other pressure sensors, is due to the contact localization of the applied force. Since the sensor works on the stress developed in the photoelastic material, the reading could be misleading if the contact is localized at one end and is detected at the centre or at the other end of the sensor. In order to improve the sensor on this account, more optical input/output fibers can be used throughout the specified breadth of the sensor and the resulting signal should be averaged-out and calibrated accordingly. The range of the applied force can be improved by increasing the contact area of the sensor, however, for many applications area enlargement may not be allowed. The other possibility is to change the photoelastic material from polyurethane to acrylic or epoxy to provide higher force range [28]. Further for compactness, the optical fibers can be discarded and U-shaped combined emitterreceiver package (typically used for end of tape detection) could be used.

\section{Discussion and conclusions}

In this paper mechanism of slip has been investigated to develop a sensor on the principles of photoelasticity for providing continuous signal during slip. The paper presented theoretical aspect as well as the experimental results of the developed sensor. The theoretical model helps identify various parameters involved in the sensor design. The sensor is novel in the sense that it provides information on slip as well as normal force with a significant improvement of delivering continuous signal during slip. Such sensors could be advantageous for grasping and manipulation tasks since they can extract slip and applied force information from a single contact location. The sensor is capable of detecting fine scratches available on the object surface, which can be exploited for quality assessment of object surfaces. Besides this, the overall size of the sensor is so small that it can be easily incorporated into robotic fingers. The top metal- lic surface of the sensor is wear resistant which can be an added advantage from an application point of view. It offers immunity to the external mechanical vibrations which has the potential for use in robotics and prosthetic applications.

As the sensor works on the change in the coefficient of friction between the sensor surface and the slipping object, the slip signal in case of some materials may be more pronounced than others. Particularly the metallic objects are expected to provide stronger signals as compared to the non-metallic objects since the variation of the coefficient of friction due to relative velocity is higher in metal-metal contacts as compared to that in metal-non-metal combinations. While attempting to improve the sensor compactness, the sensor design was changed to incorporate the emitter and the detector directly into a U-slot with the photoelastic layer. The new design was however found to be unworkable. This was due to the available size of emitter and detector which had a larger diameter $(6 \mathrm{~mm})$ as compared to the optical fibers diameter $(2 \mathrm{~mm})$ used in the earlier experiment. When these optoelectronic devices were placed directly into the U-slot, the center for signal detection was shifted away from the top surface. Since slip is a surface phenomenon, the effect of object slip far away from the surface was very much diminished. As a result, the stress change in photoelastic material due to vibration was not received at the detector. The application however, would improve if the emitter detector of smaller size (1-2 mm) can be directly placed closed to the slipping surface.

The presented design is compact, simple and yet robust as compared to the other existing designs. We are currently researching on creating a photoelastic fingertip with integrated emitter and detector pair and in-built polarizer and analyzer to investigate how the system parameters affect the sensor behavior. Even though the sensor provides different signals for different materials and slip rates, the signals from the sensor can be intelligently processed [29] to obtain useful information for grasping and manipulation tasks. It is our belief that an integrated system will be viable for a number of applications in robotics and prosthetics.

\section{Acknowledgments}

The Faculty of Engineering \& Applied Science, Department of Electrical Engineering of University of Southampton, and the Overseas Research Student award from the UK Committee of Vice-Chancellors and Principals have supported this research.

\section{References}

[1] T. Yoshikawa, Y. Yokokohji, A. Nagayama, Object handling by threefingered hands using slip motion, in: Proceedings of the IEEE/RSJ International Conference on Intelligent Robots and Systems, 1993, pp. 99-105.

[2] R.W. Todd, J.M. Nightingale, Adaptive prehension control for a prosthetic hand, in: Third International Symposium on External Control of Human Extremities, 1969.

[3] R.D. Howe, Tactile sensor for localizing transient events in manipulation, in: Proceedings of the IEEE International Conference on Robotics and Automation, 1994, pp. 471-476.

[4] M.R. Cutkosky, J.M. Hyde, Manipulation control with dynamic tactile sensing, in: Proceedings of the Sixth ISRR, Pennsylvania, 1993. 
[5] B. Magnussen, T. Doersam, The Karlsruhe dextrous hand: a three fingered robot gripper, in: Proceedings of the European Control Conference, Rome, 1995.

[6] R.M. Crowder, Sensors, touch force and torque measurement, in: R.C. Dorf (Ed.), International Encyclopedia of Robotics, John Wiley \& Sons, New York, 1988, pp. 1544-1563.

[7] R.D. Klafter, T.A. Chmielewski, M. Negin, Robotic Engineering: An integrated approach, Prentice Hall International, 1989.

[8] J.A. Dominguez-Lopez, R.I. Damper, R.M. Crowder, C.J. Harris, Adaptive neurofuzzy control of a robotic gripper with online machine learning, Robot. Autonom. Syst. 48 (2004) 93-110.

[9] P.J. Kyberd, P.H. Chappell, Characterization of an optical and acoustic touch and slip sensor for autonomous manipulation, Meas. Sci. Technol. 3 (10) (1992) 969-975.

[10] F. Eghtedari, C. Morgan, A novel tactile sensor for robot applications, Robotica 7 (1989) 289-295.

[11] K. Nishihara, S. Komiya, N. Okuma, H. Otsuka, Slip sensor using a piezoelectric bimorph element, Trans. JSME C 62 (598) (1996) 2244-2249.

[12] E.G.M. Holweg, H. Hoeve, W. Jongkind, L. Marconi, C. Melchiorri, C. Bonivento, Slip detection by tactile sensors: algorithm and experimental results, in: Proceedings of the IEEE Interantional Conference on Robotics and Automation, 1996, pp. 3234-3239.

[13] D.P.J. Cotton, A. Cranny, N.M. White, P.H. Chappell, S.P. Beeby, Design and development of integrated thick-film sensors for prosthetic hands, in: Proceedings of the Seventh Biennial Conference on Engineering Systems Design and Analysis, vol. 3, 2004, pp. 573-589.

[14] K. Kyung, S. Son, D. Kwon, M. Kim, Design of an integrated tactile display system, in: Proceedings of the IEEE International Conference on Robotics and Automation, vol. 1, 2004, pp. 776-781.

[15] J. Engel, J. Chen, C. Liu, B.R. Flachsbart, J.C. Selby, M.A. Shannon, Development of polyimide-based flexible tactile sensing skin, in: Materials Research Society Symposium Proceedings, vol. 736, 2002, pp. $165-170$.

[16] Y. Yamada, I. Fujimoto, T. Morizono, Y. Umetani, T. Maeno, D. Yamada, Development of artificial skin surface ridges with vibrotactile sensing elements for incipient slip detection, in: IEEE International Conference on Multisensor Fusion and Integration for Intelligent Systems, 2001, pp. 251-257.

[17] K. Sasaki, T. Hirota, Y. Fujikake, H. Nakaki, Signal processing for slip and contact sensing and its application to a two-fingered robotic hand, Integr. Comput. Aided Eng 8 (n4) (2001) 283-291.

[18] D. De Rossi, Artificial tactile sensing and haptic perception, Meas. Sci. Technol. 2 (1991) 1003-1016.

[19] M.R. Tremblay, W.J. Packard, M.R. Cutkosky, Utilising sensed incipient slip signals for grasp force control, in: Proceedings of Japan-USA Symposium on Flexible Automation, Part 1, 1992, pp. 1237-1243.

[20] M.R. Tremblay, M.R. Cutkosky, Estimating friction using incipient slip sensing during a manipulation task, in: Proceedings of the IEEE International Conference on Robotics and Automation, 1993, pp. 429-434.

[21] S.P. Timoshenko, J.M. Gere, Mechanics of Materials, Van Nostrand Reinhold Company, 1972.
[22] R.B. Heywood, Photoelasticity for Designers, Pergamon Press, London, 1969.

[23] E. Rabinowicz, Friction and Wear of Materials, John Wiley and Sons, 1965.

[24] V.N. Dubey, Sensing and control within a robotic end effector, Ph.D. Thesis, University of Southampton, UK, 1997.

[25] F. Eghtedari, S.H. Hopkins, D.T. Pham, Model of a slip sensor, in: Proceedings of Institute of Mechanical Engineers, part B, vol. 207, 1993 , pp. $55-64$.

[26] V.N. Dubey, R.M. Crowder, Grasping and control issues in adaptive end effectors, in: ASME International Design Engineering Technical Conference, DETC2004-57126, Salt Lake City, UT, USA, September 28-October 2, 2004.

[27] R.M. Crowder, V.N. Dubey, P.H. Chappell, Dynamic control of fingertip force using tactile sensors and fuzzy logic, in: Sixth International Conference on Control, Automation, Robotics and Vision, Singapore, 2000.

[28] S.C. Jacobsen, J.E. Wood, D.F. Knutti, K.B. Biggers, in: D.T. Pham, W.B. Heginbotham (Eds.), The Utah/MIT dextrous hand: work in progress, Robot Grippers, 1986, pp. 341-389.

[29] V.N. Dubey, R.M. Crowder, P.H. Chappell, Optimal object grasp using tactile sensors and fuzzy logic, Robotica 17 (6) (1999) 685-693.

\section{Biographies}

Venketesh Dubey received his BE and ME degrees in mechanical and design engineering from the MNR Engineering College (now MNNIT), Allahabad (India) in 1988 and 1990, respectively. He started his career as a project engineer in the area of CAD/CAM at the Indian Institute of Technology, Kanpur. He briefly held the position of scientist in robotics at the Central Mechanical Engineering Research Institute, Durgapur before moving to the MNR Engineering College as a lecturer in mechanical engineering. He then joined the University of Southampton (UK) for pursuing $\mathrm{PhD}$ where he received his doctorate degree in robotics in 1997. Subsequently he worked as a Research Fellow at the King's College London and as a lecturer at the University of Wolverhampton. He is currently a senior lecturer in engineering design at the Bournemouth University. His research interests include tactile sensing, multi-fingered robot hands, intelligent control, computer graphics and medical robotics. He is a member of the IEE and a chartered engineer.

Richard Crowder is currently senior lecturer in robotics and control in the School of Electronics and Computer Science, University of Southampton. He joined the Intelligence, Agents, Multimedia Group to develop his interests in the industrial applications of knowledge technologies. He obtained his $\mathrm{PhD}$ in electrical engineering from the University of Leicester in 1977. Prior to joining the University of Southampton he worked in the machine tool industry, specialising in high-performance systems for the aerospace industry. He has additional research interests in biologically inspired robotics, with particular reference to manipulation. Richard has authored approximately 90 refereed journal and conference papers. He has supervised a number of successful $\mathrm{PhD}$ candidates. He is a chartered engineer and a member of the Institution of Electrical Engineers. 